\title{
Scattering properties of envelope solitons in disordered systems: decay of localization effects by strong nonlinearity
}

\author{
Yuri S Kivshar†§, Sergei A Gredeskul $† \|$, Angel Sánchez $\ddagger$ and Luis Vázquez $\ddagger$ \\ $\dagger$ Institute for Low Temperature Physics and Engineering, 47 Lenin Avenue, \\ Kharkov 310164, Ukraine \\ ¥Departamento de Física Teórica I, Facultad de Ciencias Físicas, Universidad Complutense, \\ E-28040 Madrid, Spain
}

Received 25 February 1991

\begin{abstract}
We study the scattering of nonlinear wavepackets in the form of envelope solitons by a one-dimensional disordered system. We briefly review the features of the scattering for linear waves and obtain some results for linear wavepackets to demonstrate their common exponential decay of the transmission coefficient, characterized by a localization length. We consider the same process for envelope solitons, and we show in the framework of the simplest model, that, above a certain threshold, strong nonlinearity allows undistorted propagation of these wavepackets. We describe how this behaviour can be obtained, for the nonlinear Schrödinger equation, by means of a simple independent scattering approach, using results of soliton perturbation theory to compute one-impurity reflection coefficients in the Born approximation. We derive equations to describe the transmission of the soliton parameters and we analyse them in full detail. The main result of our study is the conclusion that a strong nonlinearity stipulates two spatial scales in the wavepacket scattering by disordered systems. The first spatial scale depends on the amplitude and it may be very large, and the second one is the usual localization length. As a consequence the nonlinear wavepackets or solitons are much more stable against disorder than linear ones.
\end{abstract}

\section{Introduction}

In the last few years nonlinear systems have increasingly attracted researchers from different areas. In particular, the problem of wave propagation in nonlinear disordered media has received considerable attention [1-7] because of its many applications not only in condensed matter physics but also in other very active fields. The main question that this investigation addresses concerns the simultaneous competition of disorder and nonlinearity on the wave dynamics, which may lead to complex properties in the system under consideration. Thus, while disorder in one-dimensional linear systems usually originates Anderson localization (e.g. see [8]), a phenomenon that is easily generalized to other waves like phonons, acoustic and electromagnetic waves, and so on, it has been found that nonlinearity may change the dynamics of these systems and give rise to qualitatively new effects. This is the case of the celebrated result that weak nonlinearity acting against disorder changes the length dependence of the transmission coefficient:

$\S$ Present address: Institut für Theoretische Physik I, Heinrich-Heine-Universität Düsseldorf, D-4000 Düsseldorf 1, Federal Republic of Germany.

\| Present address: Department of Physics, Ben-Gurion University of the Negev, PO Box 84105, Beer Sheva, Israel. 
it still tends to zero as the size of the system increases, but following a power law (that can be either $L^{-1 / 2}$ or $L^{-3 / 2}$, see $[1,2,4]$ ) instead of the exponential one proper to the linear equation.

The previous results apply to a nonlinear plane wave when transmitted through a medium governed by the reduced, time-independent nonlinear Schrödinger (NLS) equation. Nevertheless, it is well known that in the nonlinear system with 'focusing' nonlinearity plane waves are modulationally unstable to formation of sidebands (the so-called Benjamin-Feir instability) and, as a consequence, they decay into localized objects, namely nonlinear wavepackets or solitons. Modulational instability is forced by disorder $[9,10]$, and, as a result, a nonlinear plane wave decays into a set of solitons much faster than in the homogeneous case [11]. In pure (homogeneous) systems, solitons propagate without suffering changes in their shape or velocity, and their interactions result in phase shifts only. Attending to their properties, this kind of excitation is generally classified into three general types: dynamical solitons (and kinks), topological kinks and envelope solitons. Solitons belonging to the first class, i.e. dynamical solitons and dynamical (non-topological) kinks, have an amplitude proportional to their velocity, and their scattering in a disordered system is similar to that of a linear wavepacket [3]. The next type of solitary wave are topological kinks, which can only exist in nonlinear systems with two or more equivalent (or almost equivalent) ground states; in a number of situations, kink dynamics may be described by equations for an effective (classical or relativistic) particle, the particle coordinate being the kink position (e.g. see [12]). Subsequently, it turns out that topological kinks are less scattered by disordered systems than dynamical ones [7]. So far, these first two kinds of nonlinear excitations share the fact that they depend only on one parameter. The other one, envelope solitons, are two parametrical and demonstrate much more complex behaviour. Thus, as was first predicted in [6], strong nonlinearity may compensate localization effects and the propagation of nonlinear wavepackets as solitary waves (envelope solitons) in disordered systems of rarified point impurities may be allowed in an almost undistorted form; it was also proved that there exists a threshold in the soliton amplitude for this to happen. This paper is based in our letter [6] and aims to present detailed and extended results related to the scattering of envelope solitons in disordered systems. We also present new results about the scattering of linear wavepackets to compare them with the scattering of nonlinear pulses of similar shapes.

To study the influence of nonlinearity on the properties of disordered systems we cannot use the previously developed techniques, which are suitable for linear systems [8]. So, we investigate the influence of nonlinearity on disorder from another viewpoint, considering essentially nonlinear, powerful perturbative procedures. In such an approach the main assumption that we make is that during the scattering the nonlinear wavepacket exists as a localized object with changing parameters; this persistence of the soliton is the very origin of the nonlinear character of the techniques that we use.

The paper is organized as follows. Section 2 describes our model and presents the problem that we are going to deal with. In section 3 we briefly review the scattering of linear waves and obtain some new results for linear wavepackets in a disordered medium. These results are important to compare them to the outcomes of nonlinear scattering, especially for the same envelope of the nonlinear wavepacket. Analytical results based on the soliton perturbation theory and the independent scattering approach are obtained in section 4. In the same section we use numerical calculations to analyse the behaviour of the soliton parameters described by the effective equations derived in the framework or the mentioned approximations. Our predictions about general properties of nonlinear 
wavepacket scattering in disordered systems are contained in section 5 , as well as a short discussion on the physics underlying our findings.

\section{Model}

We start from the nonlinear Klein-Gordon equation for the wave variable $U(x, t)$,

$$
U_{t t}-U_{x x}+\omega_{0}^{2} U-\beta U^{3}=0
$$

$\omega_{0}$ being the frequency in the linear limit. Equation (1) arises in a number of different physical problems (e.g. see [13-16]), and it may also be obtained as an expansion of the widely applicated sine-Gordon equation with $U \ll 1$. In the framework of equation (1) we will consider the effects of the appearance of disorder related to changes of the frequency, in the form of random point impurities with intensities $\varepsilon_{n}$ and random positions $x_{n}$ :

$$
\omega_{0}^{2} \rightarrow \omega_{0}^{2}+\varepsilon(x) \quad \varepsilon(x) \equiv \sum_{n} \varepsilon_{n} \delta\left(x-x_{n}\right) .
$$

With this change, equation (1) takes the form

$$
U_{t t}-U_{x x}+\omega_{0}^{2} U-\beta U^{3}=-\varepsilon(x) U
$$

and may be thought of as a perturbed nonlinear Klein-Gordon equation. To study the properties of nonlinear wavepackets in equation (3), we will obtain an equation for an envelope $\tilde{u}(x, t)$, assuming

$$
U(x, t)=\tilde{u}(x, t) \mathrm{e}^{\mathrm{i} \omega_{0} t}+\tilde{u}^{*}(x, t) \mathrm{e}^{-\mathrm{i} \omega_{0} t}
$$

the asterisk denoting complex conjugation. In this way we are separating the system dynamics into relatively fast oscillations with linear frequency $\omega_{0}$, and a slowly varying nonlinear envelope $\tilde{u}(x, t)$. Substitution of equation (4) into equation (3) leads, in the lowest order of the oscillations, to the equation (e.g. see [17])

$$
\tilde{u}_{t t}+2 \mathrm{i} \omega_{0} \tilde{u}_{t}-\tilde{u}_{x x}-3 \beta|\tilde{u}|^{2} \tilde{u}=-\varepsilon(x) \tilde{u} .
$$

If we assume a slowly varying $\tilde{u}$, the inequality $\tilde{u}_{t t} \ll \omega_{0} \tilde{u}_{t}$ is valid and, therefore, equation (5) may be transformed into the well-known NLs equation for the dimensionless envelope:

$$
\mathrm{i} u_{\tau}+u_{x x}+2|u|^{2} u=\varepsilon(x) u
$$

where

$$
\tau=-\frac{t}{2 \omega_{0}} \quad u=\sqrt{\frac{3 \beta}{2}} \tilde{u}
$$

$\varepsilon(x)$ being the same as in equation (3).

Equation (6) at $\varepsilon=0$ is the exactly integrable NLs equation (e.g. see [17]) and, aside from the application here considered, it is important in its own right, for it has a bearing on a number of problems related to solid state physics (e.g. see [12-19]). The function $\varepsilon(x)$ may then represent, for example, the structural disorder of the associated system. The most remarkable feature of the homogeneous nonlinear system described by equation (6) at $\varepsilon(x)=0$ is that it allows the distortionless propagation of localized excitations 
in the form of envelope solitons:

$$
u_{\mathrm{s}}(x, \tau)=a \frac{\exp \left[+\mathrm{i} V x / 2-\mathrm{i}\left(V^{2} / 4-a^{2}\right) \tau\right]}{\cosh [a(x-V \tau)]}
$$

where $a$ and $V$ are the soliton amplitude and velocity, respectively. These are the objects we are interested in, and whose propagation along the disordered medium for $\varepsilon(x) \neq 0$ we intend to describe in the following.

\section{Scattering of linear waves and wavepackets}

First, neglecting the last term in the LHS of equation (6), we will restrict ourselves to the linear case. Furthermore, we will start considering the propagation of monochromatic waves in a randomly inhomogeneous medium. The study of this subject leads to the stochastic differential equation $-u_{x x}+\varepsilon(x) u=k^{2} u$, where $k$ is the wavenumber (the relation $\omega=k^{2}$ is the dispersion law in the homogeneous medium). The most important phenomenon is the localization of states by random inhomogeneities due to scattering [8]. Localization implies that the transmission coefficient $T$ decays exponentially with the system length $L$. If $\varepsilon(x)$ is a stationary, ergodic random process, then it can be proved that a positive finite number exists, the so-called localization length $\lambda(k)$, such that

$$
L^{-1} \ln T(k) \sim-\lambda^{-1}(k)
$$

for large $L$ and, hence, if $L$ is significantly wider than $\lambda(k)$, it is obvious from equation (9) that very little transmission will be allowed. If, for the sake of simplicity, we set to the same value the intensities of the impurities, $\varepsilon_{n}=\varepsilon$, and introduce $p^{-1}$ as a parameter having the sense of a mean distance between impurities, the localization length for the linear Schrödinger equation has the form $[8,20]$

$$
\lambda(k)=4 k^{2} / p \varepsilon^{2}
$$

provided that the following conditions hold:

$$
\varepsilon^{2} \ll k^{2} \quad p \ll k \quad k^{2} \ll \varepsilon^{2} L p .
$$

Linear wavepackets evolving under the linear equation also show a decay of the transmission coefficient. Let us consider the scattering of the wavepacket

$$
u_{\mathrm{wp}}(x, \tau)=\int \frac{\mathrm{d} k}{2 \pi} P(k) \mathrm{e}^{\mathrm{i} k x-\mathrm{i} k^{2} \tau}
$$

in the system of disordered point impurities, where $P(k)$ has the sense of the spectral function of the wavepacket. To compare subsequent results with the scattering of the soliton (8) in the same system, we have to choose the function $P(k)$ in the form

$$
P(k)=\frac{\pi \mathrm{i}}{\cosh \left[(\pi / 2 a)\left(k-k_{0}\right)\right]}
$$

the parameter $k_{0} \equiv V / 2$ being the carrier wavenumber of the wavepacket. The reason for making this choice is that the soliton (8), in the limit $a \rightarrow 0$ (small-amplitude limit), exhibits the same shape described by the formulae (12) and (13). 
The transmission coefficient of the wavepacket (12) may be presented in the form

$$
T_{\mathrm{wp}}=\frac{\int_{-\infty}^{\infty} \mathrm{d} k T(k)|P(k)|^{2}}{\int_{-\infty}^{\infty} \mathrm{d} k|P(k)|^{2}} .
$$

If we compute the integral in the denominator of equation (14), inserting the function $P(k)$ given by equation (13), we have the expression

$$
T_{\mathrm{wp}}=\frac{\pi}{4 a} \int_{-\infty}^{\infty} \mathrm{d} k T(k) \operatorname{sech}^{2}\left(\frac{\pi}{2 a}\left(k-k_{0}\right)\right) .
$$

Using the result (15) we may define the mean value $\left\langle T_{\mathrm{wp}}\right\rangle$ for the whole packet as follows:

$$
\mathscr{T} \equiv\left\langle T_{\mathrm{wp}}\right\rangle=\frac{\pi}{4 a} \int_{-\infty}^{\infty} \mathrm{d} k\langle T(k)\rangle \operatorname{sech}^{2}\left(\frac{\pi}{2 a}\left(k-k_{0}\right)\right)
$$

where

$$
\langle T(k)\rangle \equiv \frac{\pi^{5 / 2}}{2}\left(\frac{L}{\lambda(k)}\right)^{-3 / 2} \exp \left(-\frac{L}{4 \lambda(k)}\right)
$$

is the mean transmission coefficient with $\lambda(k)$ defined in equation (10), and under the restrictions (11) (see details in, for example, $[8,20]$ ). Further calculations yield the following results for the mean transmission coefficient $\mathscr{T}$ :

$$
\mathscr{T}= \begin{cases}\left\langle T\left(k_{0}\right)\right\rangle\left(1+\frac{1}{6} \xi^{2}\right) & \text { if } \xi \ll 1 \\ \frac{\pi^{2} a}{4 \sqrt{3} k} \exp \left(-\frac{3 \pi}{2 a} \bar{k}\left(1-\frac{2}{3} \xi^{-1 / 3}\right)\right) & \text { if } \xi \gtrsim 1\end{cases}
$$

where we have noted

$$
\xi \equiv\left(\frac{\bar{k}}{k_{0}}\right)^{3} \quad \bar{k} \equiv\left(\frac{L \varepsilon^{2} a p}{2 \pi}\right)^{1 / 3} .
$$

The meaning of $\xi$ and $\bar{k}$ is the following: the parameter $\xi$ describes corrections to the trivial result in equation (18), and the parameter $\bar{k}$ has the sense of the characteristic wavenumber stipulated by the disorder (see equation (19)). From equation (18), it can be seen that when $\xi \ll 1$ the mean transmission coefficient is defined by the contributions of wavenumbers in the vicinity of $k=k_{0}$; on the other hand, when $\xi \gtrsim 1$, this value is defined by the wavenumbers near the point $k=\bar{k}$. In both of these cases conditions (11) must be valid for their respective characteristic values of $k$.

Another important characteristic scattering parameter is the transmission coefficient defined for a unique realization of the random process. This transmission coefficient for a plane wave may be also defined asymptotically and has the form of equation (17) with the modified exponential multiplier $\exp (-L / \lambda(k))$. Using the asymptotic relation (9), the transmission coefficient of the wavepacket can be calculated (within logarithmic accuracy) starting from formula (15), and we obtain

$$
\ln T_{\mathrm{wp}} \simeq \begin{cases}\ln T\left(k_{0}\right) & \text { if } \xi \ll 1 \\ -\frac{3 \pi}{a \sqrt[3]{2}} \exp \left[\bar{k}\left(1-\frac{\sqrt[3]{2}}{3} \xi^{-1 / 3}\right)\right] & \text { if } \xi \gtrsim 1\end{cases}
$$


where $\xi$ and $\bar{k}$ are defined in equation (19). The formulae are very similar to equations (18). Therefore, it is clear that the general behaviour of the wavepacket scattering described by the mean transmission coefficient $\mathscr{T}=\left\langle T_{\text {wp }}\right\rangle$ or the transmission coefficient $T_{\mathrm{wp}}$ defined on a realization is qualitatively the same and also qualitatively coincide with that of a plane (single) wave, i.e. an exponential decay of the transmission coefficient in the linear system characterizes both a plane wave and a wavepacket.

\section{Soliton scattering in a disordered system}

\subsection{Independent scattering approach}

We next move to the nonlinear problem, i.e. the scattering of the soliton (8) by a random system of point impurities with equal intensities $\varepsilon$. The soliton is incident on the disordered layer from the left, scatters, and decomposes into reflected $(r)$ and transmitted $(t)$ parts. We assume that after passing through each impurity the wavepacket may be presented again as a soliton plus some small-amplitude waves (radiation). To begin our approach to the calculation, we recall that the NLS system is characterized by two integrals of motion, namely the energy $E$ and the 'number of quasi-particles' $N$ defined by the standard expressions

$$
\begin{aligned}
& E=\int_{-\infty}^{\infty} \mathrm{d} x\left(\left|u_{x}\right|^{2}+\varepsilon(x)|u|^{2}-|u|^{4}\right) \\
& N=\int_{-\infty}^{\infty} \mathrm{d} x|u|^{2}
\end{aligned}
$$

During the scattering the values of these quantities defined for the soliton, will change due to the emission of radiation, but they are conserved for the total system. So, we describe the scattering process through two magnitudes: the total energy transmission coefficient $T^{(E)}=E_{\mathrm{t}} / E_{\mathrm{i}}$, that is, the transmitted energy $E_{\mathrm{t}}$ over the incident energy $E_{\mathrm{i}}$, and the 'number of particles' transmission coefficient $T^{(N)}=N_{\mathrm{t}} / N_{\mathrm{i}}, N_{\mathrm{t}}$ and $N_{\mathrm{i}}$ defined analogously. It is to be understood that the constraints $E_{\mathrm{i}}=E_{\mathrm{t}}+E_{\mathrm{r}}=$ const and $N_{\mathrm{i}}=N_{\mathrm{t}}+N_{\mathrm{r}}=$ const must hold.

When the concentration $p$ of impurities is low, it is quite reasonable to suppose that the average distance between two neighbouring impurities is larger than the soliton size. In this limit we may treat the scattering by many impurities independently, defining a transmission coefficient for the whole layer as $T \approx \prod_{j} T_{j}, T_{j}$ being the transmission coefficient of the $j$ th impurity. In this so-called independent scattering approach, backscattering interference effects are neglected, and hence the transmitted soliton for the $j$ th impurity is then the incident one for the $(j+1)$ th scatterer, and we can write (cf. $[3,6])$

$$
\begin{aligned}
& E_{j+1}=E_{j} T_{j}^{(E)}\left(E_{j}, N_{j}\right) \\
& N_{j+1}=N_{j} T_{j}^{(N)}\left(E_{j}, N_{j}\right)
\end{aligned}
$$

and

$$
\begin{aligned}
& \Delta E_{j+1}=E_{j+1}-E_{j}=-E_{j} R_{j}^{(E)}\left(E_{j}, N_{j}\right) \\
& \Delta N_{j+1}=N_{j+1}-N_{j}=-N_{j} R_{j}^{(N)}\left(E_{j}, N_{j}\right)
\end{aligned}
$$


where $R_{j}^{(E, N)}=1-T_{j}^{(E, N)}$ stand for energy and 'number of particles' reflection coefficients. It is very important to note that, unlike the linear case, in the nonlinear problem we have to distinguish two independent characteristics of the scattering, e.g. two reflection coefficients. Since on the average there are $(\Delta x) p$ impurities in the interval $\Delta x$, we can derive from equations (25) and (26) the following differential equations:

$$
\begin{aligned}
& \frac{\mathrm{d} E}{\mathrm{~d} x}=-p E(x) R^{(E)}(E(x), N(x)) \\
& \frac{\mathrm{d} N}{\mathrm{~d} x}=-p N(x) R^{(N)}(E(x), N(x))
\end{aligned}
$$

assuming that during the scattering the soliton parameters have small changes. In order to deal with the system (27) and (28), it is now necessary to compute the exact soliton reflection coefficients $R^{(N)}(E, N)$ and $R^{(E)}(E, N)$ defined for a single inhomogeneity. We will address this point in the next subsection; to finish this one, we must stress the fact that, in spite of their aspect, equations (27) and (28) still conserve the influence of randomness, through the use of mean transmission coefficients and a mean 'number of impurities in the interval'. Other choices for the spatial array of the impurities, as, for instance, a periodic lattice (like the one studied in [21]), could not be well described by these equations, because of correlations between lattice spacing and width of the propagating soliton or coherence phenomena.

\subsection{Soliton reflection coefficients}

Soliton reflection coefficients may be calculated via soliton perturbation theory (e.g. see $[12,22])$. The reflection coefficients are defined by the reflected wavepackets in the form of linear waves emitted by the soliton during the scattering. The inverse scattering transform (IST) gives the corresponding expression for the spectral density of emitted waves (see details in [12]),

$$
n_{\mathrm{rad}}(\lambda, \tau)=\frac{1}{\pi}|b(\lambda, \tau)|^{2} \quad \text { at }|b(\lambda, \tau)|^{2} \ll 1
$$

$b(\lambda, \tau)$ being the so-called Jost coefficient related to the IST for the NLS equation. The spectral parameter $\lambda$ appearing in the IST is connected with the wavenumber $k(\lambda)$ and the frequency $\omega(\lambda)$ of generated linear waves by the relations $\omega(\lambda)=k^{2}(\lambda)=4 \lambda^{2}$. The influence of a perturbation $P(u)$ leads to a change of the IST spectral coefficients that also affects the Jost coefficient $b(\lambda, \tau)$, which can be written in the form of the equation (e.g. see [12])

$$
\begin{aligned}
\frac{\partial b(\lambda, \tau)}{\partial \tau}=4 \mathrm{i} \lambda^{2} b(\lambda, \tau)+\varepsilon \int_{-\infty}^{\infty} \mathrm{d} x\left(P(u) \Phi_{1}^{(1)}(x, \tau ; \lambda) \Phi_{2}^{(2)}(x, \tau ; \lambda)\right. \\
\left.-\quad P^{*}(u) \Phi_{2}^{(1)}(x, \tau ; \lambda) \Phi_{1}^{(2)}(x, \tau ; \lambda)\right) \\
\begin{aligned}
\frac{\partial b(\lambda, \tau)}{\partial \tau}=4 \mathrm{i} \lambda^{2} b(\lambda, \tau)+\frac{\varepsilon}{\left[(\lambda+V / 2)^{2}+a^{2} / 4\right]} \\
\times\left[\frac{a^{2}}{4} \int_{-\infty}^{\infty} \mathrm{d} x P\left(u_{\mathrm{s}}\right) \frac{\exp (-2 \mathrm{i} \lambda x-\mathrm{i} V x+2 \mathrm{i} \Delta(\tau))}{\cosh ^{2} Z}\right. \\
\left.-\int_{-\infty}^{\infty} \mathrm{d} x P^{*}\left(u_{\mathrm{s}}\right) \mathrm{e}^{-2 \mathrm{i} \lambda x}\left(\lambda+\frac{V}{4}-\frac{\mathrm{i} a}{2} \tanh Z\right)^{2}\right]
\end{aligned}
\end{aligned}
$$


where $Z=a(x-V \tau)$ and $\Delta=\left(V^{2} / 4-a^{2}\right) \tau$. To describe the effects of a single impurity we have to use the perturbation $P(u)$ in the form $\varepsilon \delta\left(x-x_{0}\right) u, x_{0}$ being the position of the impurity.

If before the scattering the wave is a soliton, described by equation (8), the initial condition for equation (31) should be taken in the form $b(\lambda, \tau=-\infty)=0$. Having integrated this equation, one can find the radiative density after the scattering with the help of equation (29):

$$
n_{\mathrm{rad}}(\lambda, \tau=+\infty)=\frac{1}{\pi}|b(\hat{\lambda}, \tau=+\infty)|^{2} .
$$

Expression (32) allows us to calculate the 'number of quasi-particles' and the energy of reflected waves, which are given by

$$
N_{\mathrm{r}}=\int_{-\infty}^{0} \mathrm{~d} \lambda n_{\mathrm{rad}}(\lambda) \quad E_{\mathrm{r}}=4 \int_{-\infty}^{0} \mathrm{~d} \lambda \hat{\lambda}^{2} n_{\mathrm{rad}}(\lambda)
$$

and to define the soliton reflection coefficients

$$
R^{(N)}=\frac{N_{\mathrm{r}}}{N} \quad R^{(E)}=\frac{E_{\mathrm{r}}}{E} .
$$

Here $N$ and $E$ are the 'number of particles' and the energy corresponding to the single unperturbed soliton (8):

$$
N=2 a \quad E=\frac{1}{4} N\left(V^{2}-\frac{1}{3} N^{2}\right) .
$$

The exact results may be obtained in the Born approximation which is valid for small $\varepsilon$ and $V^{2} \gg|\varepsilon| a$. Using expressions (8), (31), (32) and (33) it is possible to arrive at the following equations for the soliton reflection coefficients:

$$
\begin{aligned}
R^{(N)} & =\frac{\pi \varepsilon^{2}}{64 N V} \int_{0}^{\infty} \mathrm{d} y F(y, \alpha) \\
R^{(E)} & =\frac{\pi \varepsilon^{2} V}{256 E} \int_{0}^{\infty} \mathrm{d} y y^{2} F(y, \alpha)
\end{aligned}
$$

where

$$
F(y, \alpha)=\frac{\left[(y+1)^{2}+\alpha^{2}\right]^{2}}{\cosh ^{2}\left[\pi\left(y^{2}+\alpha^{2}-1\right) / 4 \alpha\right]}
$$

and $\alpha \equiv N / V$. As a consequence, equations (27) and (28) give rise to the following system of integrodifferential equations:

$$
\begin{aligned}
& \frac{\mathrm{d} N}{\mathrm{~d} z}=-\frac{1}{V} \int_{0}^{\infty} \mathrm{d} y F(y, \alpha) \\
& \frac{\mathrm{d} V}{\mathrm{~d} z}=-\frac{1}{2 N} \int_{0}^{\infty} \mathrm{d} y\left(y^{2}-1\right) F(y, \alpha)-\frac{N}{2 V^{2}} \int_{0}^{\infty} \mathrm{d} y F(y, \alpha)
\end{aligned}
$$

where the distance is measured in units of $x_{0}=64 / \pi p \varepsilon^{2}$, i.e. $z=x / x_{0}$. Notice that, for convenience, we have used relationship (35) to write down an equation for $V$ instead of the one we previously have for $E$. 


\subsection{Evolution of the soliton parameters}

In the remaining part of this section we describe the variation of $N$ and $V$ along the $z$-axis. By carefully studying the system (39) and (40), it turns out that $\alpha$ is a very relevant parameter of the problem. This quantity is physically significant because it is related to the nonlinearity of the incoming soliton; the soliton will be 'more nonlinear', so to speak, for large values of $\alpha$. This can be understood by realizing that, for each given value of $V(0)$, the greater $\alpha$ is the larger becomes the number of quasi-particles contained in the soliton and, in addition, its spatial extent becomes narrower; this is obvious from formula (8). On the contrary, if $\alpha$ is small, it can be easily seen that the wave looks very similar to a linear wavepacket (cf. equation (8) and equations (12) and (13)).

Once we have clarified the physical meaning of $\alpha$, we begin the analysis of the two coupled integrodifferential equations (39) and (40). We first consider the linear limit, $\alpha \ll 1$; in this simplification, the system (39) and (40) can be solved analytically in an quite approximate fashion, yielding (note that if $\alpha \ll 1$ the derivative of $V$ becomes negligible, for the integrals are never much greater than unity) $V(x)=V(0)=$ const and, therefore, the two transmission coefficients are the same:

$$
T^{(N, E)}(x)=N(x) / N(0)=E(x) / E(0)=\mathrm{e}^{-x / \lambda_{0}}
$$

where $\lambda_{0} \equiv V^{2}(0) / p \varepsilon^{2}=1 / p R_{1}, R_{1} \equiv \varepsilon^{2} / V^{2}(0)$ being the reflection coefficient of a single impurity. This result demonstrates the exponential decay of the transmission coefficient, and it is a consequence of the quasilinear features of solitons with small $\alpha$, which closely resemble those of linear wavepackets. As we can see, these equations again show the same dependence as that in equations (9) and (18), where $\lambda\left(k_{0}\right)=\lambda_{0}$, and $k_{0}=V / 2$ has the sense of a carrier wavenumber of the packet [see equation (8)]. The last term on the RHS of (18) reflects from this viewpoint the influence of the wavepacket width $\left(a^{-1}\right)$ on the mean transmission coefficient acting against the disorder.

In the opposite limit, $\alpha \sim 1$ or greater, the system of integrodifferential equations (39) and (40) was numerically solved. A simple rectangle method was chosen to compute the integrals, with a mesh size proportional to the value of $\alpha$, and recalculated at each time step, to maintain accuracy within the required limits. With regard to the differential equations themselves, they are rather well behaved, and it was enough to employ a Euler procedure to integrate them. Nevertheless, we repeated the solutions for several initial conditions with a more precise leap-frog scheme, always finding a fairly good agreement between the results from both methods. In this way we concluded that the possible evolution of the system can be described as follows: the dynamics depends essentially on the value of the parameter $\alpha(0)=N(0) / V(0)$, because the system given by equations (39) and (40) has a fixed point for $\alpha$, namely the solution of the transcendental equation

$$
\alpha_{\mathrm{c}}^{2}-2+G\left(\alpha_{\mathrm{c}}\right)=0 \quad G(\alpha) \equiv \frac{\int_{0}^{\infty} \mathrm{d} y\left(y^{2}-1\right) F(y, \alpha)}{\int_{0}^{\infty} \mathrm{d} y F(y, \alpha)}
$$

We computed this value both by numerically solving this equation and by direct integration of the system (39) and (40), obtaining perfect concordance between both results, and concluding that $\alpha_{c}=1.28505(4)$. Further, as is shown in the appendix, we have also found that $\alpha_{c}$ is an unstable fixed point. This fact allows us to be confident in the analysis we report below, which will remain true no matter how close we are to $\alpha_{c}$. 
A straightforward calculation of the derivative of $\alpha(z)$ is the only requirement to establish that only two kinds of behaviour are allowed for the system (39) and (40):

(i) $\alpha(0)<\alpha_{c}$. The system evolves to a final state in which $N$ tends asymptotically to zero while $V$ goes to a constant, non-zero value, and hence $\alpha(\infty)=0$, as imposed by the above-mentioned considerations. This behaviour corresponds to a decay of the transmission coefficient and, as we have seen before, for small enough $\alpha$ this decay is exponential and is quite correctly described by formula (41) (see figure 1). If $1 \sim \alpha<\alpha_{c}$ the decay of $N(z)$, though still present, is more complicated: it is slow at first and, after an interval, it undergoes a crossover to a fast exponential decrease, faster than that which takes place when $\alpha \ll 1$ (see figure 2).

(ii) $\alpha(0)>\alpha_{c}$. This type of initial condition leads to solutions such that both $n$ and $V$ suffer a very rapid decay at first up to a point at which the two functions become constant for practical regions. Obviously, the same happens to $\alpha$, which tends to a value of about 10 when $z$ goes to infinity. Figures $3-5$ show some features of the asymptotic dynamics concerning the dependence on the initial values of $N(0)$ or $V(0)$. Each choice of initial conditions gives rise to different shapes of $T^{(E)}(z)$ and $T^{(N)}(z)$, even for the same values of $\alpha$ (cf. figure 3 ), and we have found that the initial transients are very

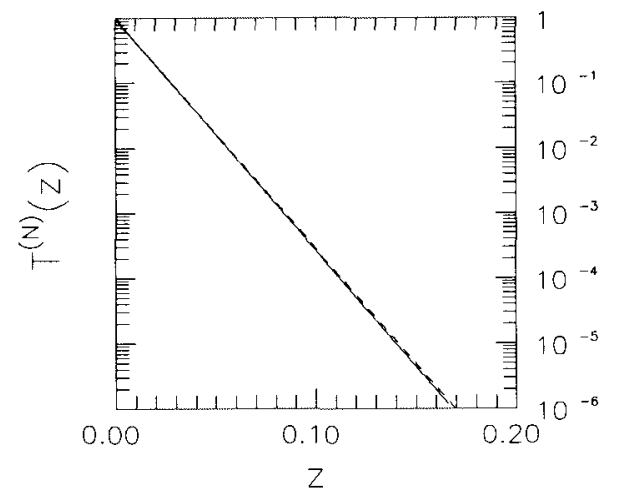

Figure 1. The transmission coefficient $T^{(N)}(z)=N(z) / N(0)$ versus $z$ when initial conditions are $N(0)=0.01$ and $V(0)=0.5(\alpha(0)=0.02)$. The full line is obtained numerically, and the broken line is the approximate analytical solution.

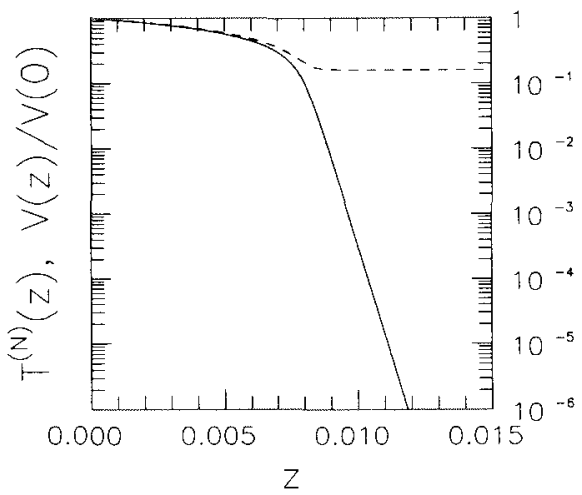

Figure 2. The transmission coefficient $T^{(N)}$ (full line) and the function $V(z) / V(0)$ (broken line) when initial conditions are $N(0)=0.625$ and $V(0)=0.5(\alpha(0)=1.25)$. 

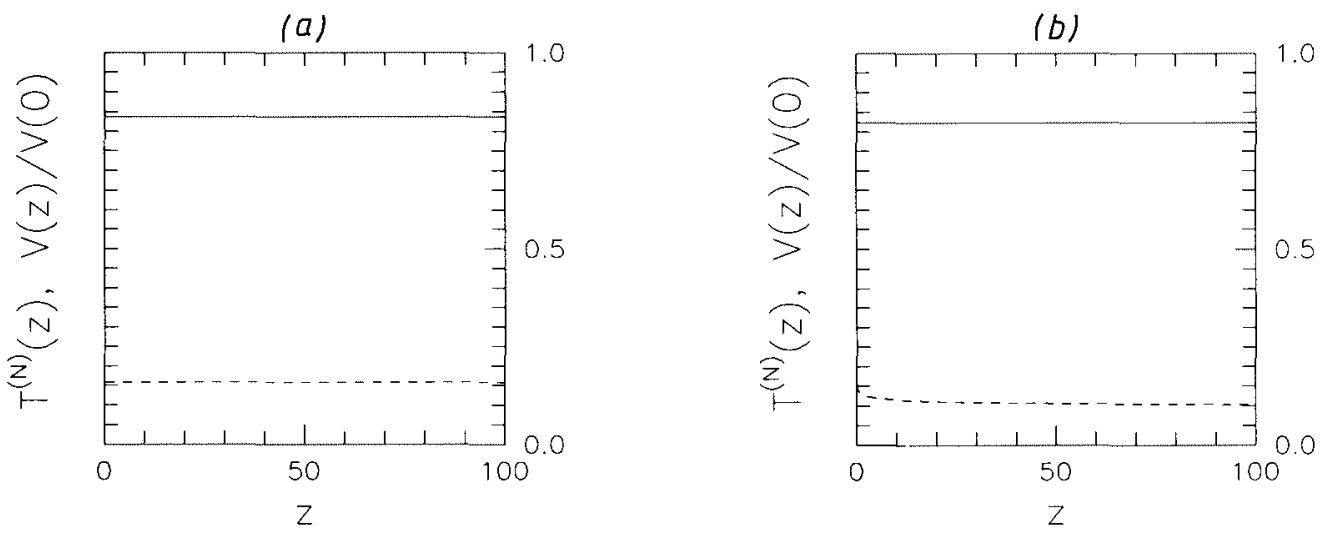

Figure 3. As figure 2 but for $(a) N(0)=0.25$ and $V(0)=0.1$ and $(b) N(0)=1.25$ and $V(0)=0.5$. In both cases $\alpha=2.5$.

sensitive to the values of $N(0)$ and $V(0)$ : the slope at small values of $Z$ is steeper for smaller values of $N(0)$ and $V(0)$, and the asymptotic regime is reached in a shorter interval. This point is illustrated in figure 6; the transient usually arises in a interval too small to be appreciated in the long scale needed to show the persistence of the propagation, and slopes can reach values up to $10^{5}$. However, the general property of these curves is the same: both transmission coefficients tend to certain asymptotic values,
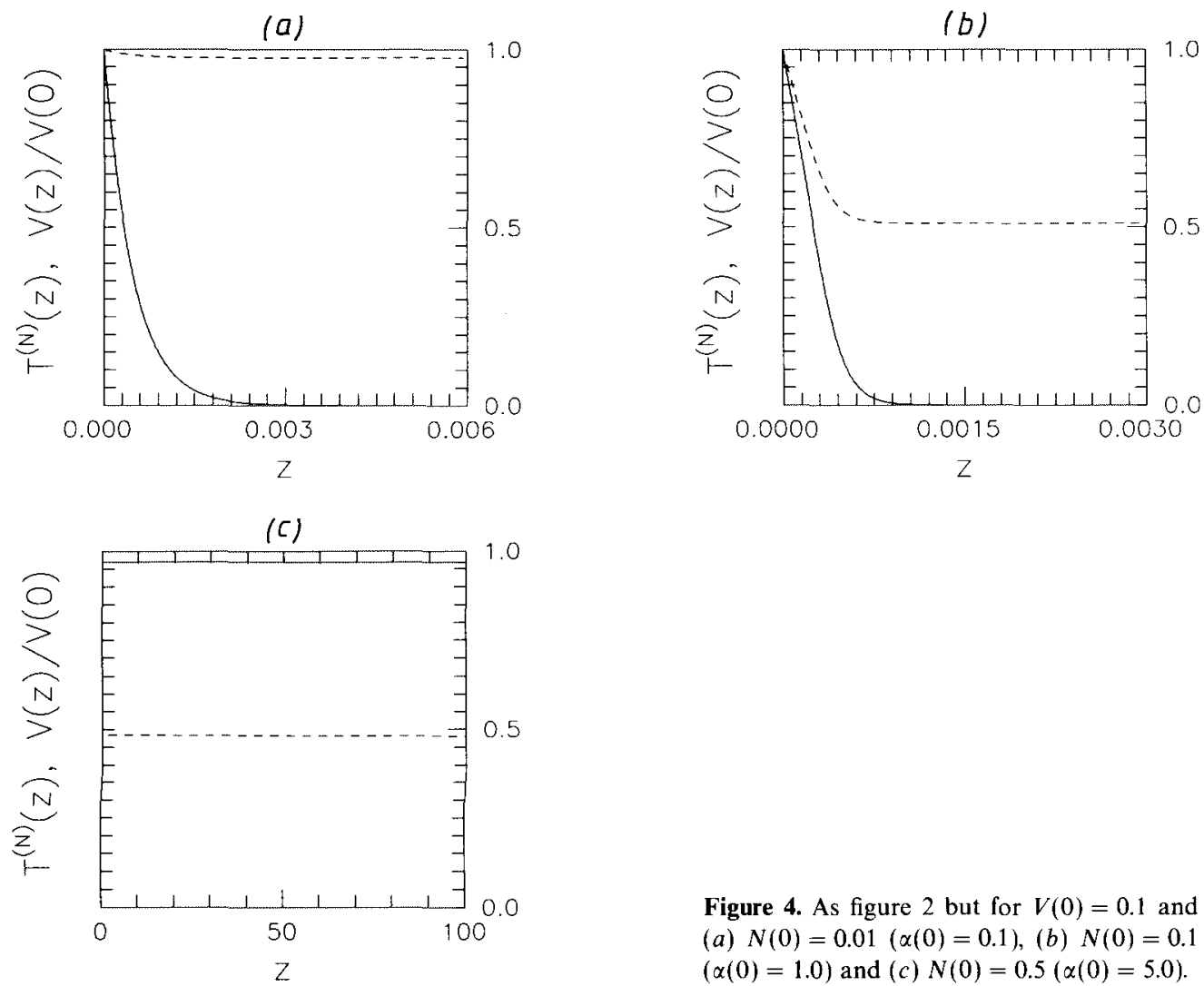

Figure 4. As figure 2 but for $V(0)=0.1$ and (a) $N(0)=0.01(\alpha(0)=0.1),(b) N(0)=0.1$ $(\alpha(0)=1.0)$ and $(c) N(0)=0.5(\alpha(0)=5.0)$. 
(a)

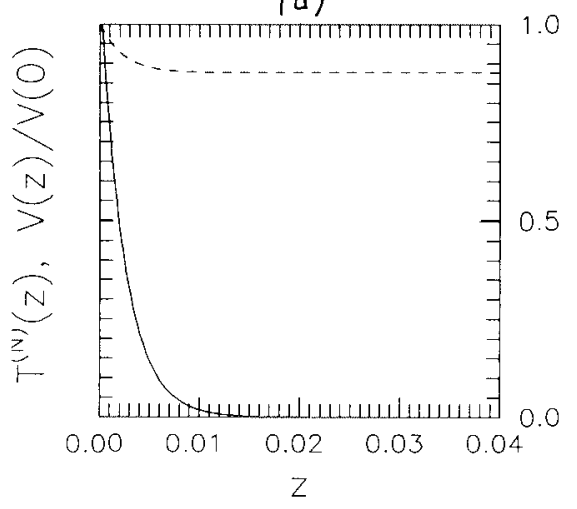

(c)

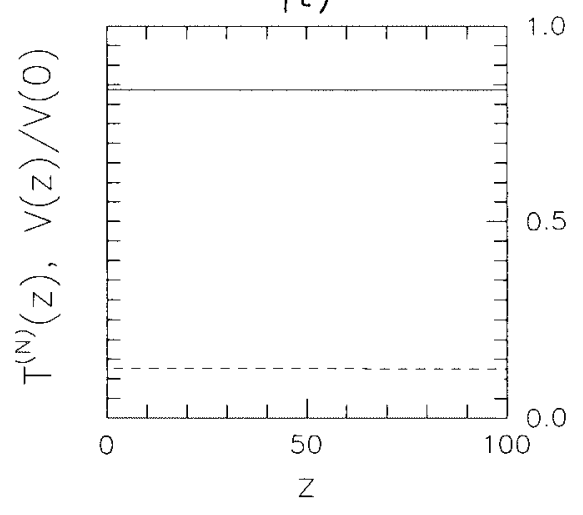

(b)

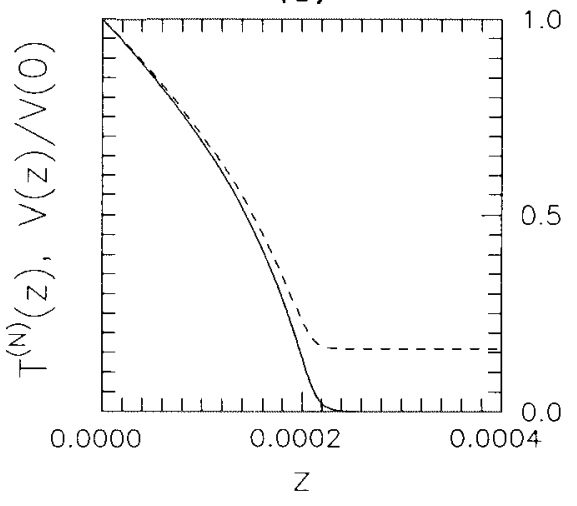

Figure 5. As in figure 2 but for $N(0)=0.1$ and (a) $V(0)=0.25(\alpha(0)=0.4),(b) V(0)=0.08$ $(\alpha(0)=1.25)$ and $(c) V(0)=0.04(\alpha(0)=2.50)$.

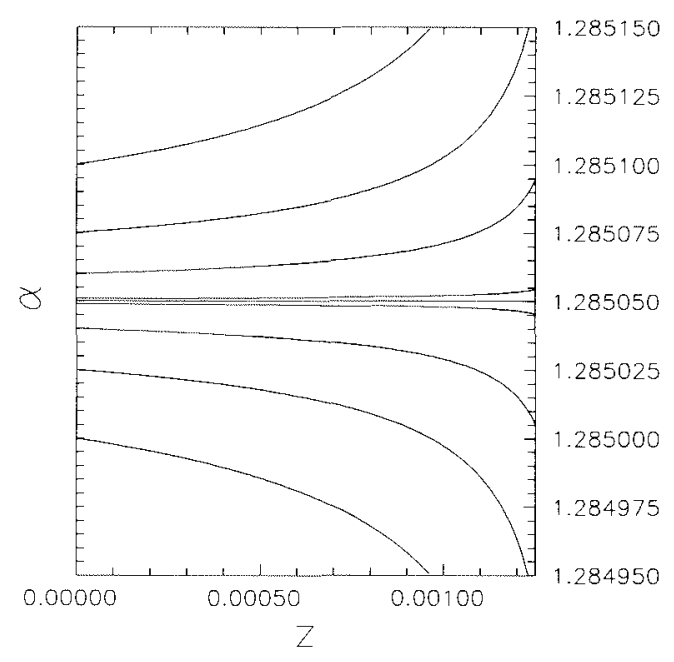

Figure 6. Several shapes of $\alpha(z)$ according to our approximate analysis in the appendix. The plots are obtained from equation (A7). 
and, henceforth, the rearranged wavepacket is transmitted without any reflection, i.e. localization is inhibited. For different sets of $N(0)$ and $V(0)$ the final dependences when they give rise to equal values of $\alpha(0)$ are almost the same (see figures 4 and 5).

\section{Concluding remarks}

In conclusion, we have considered the NLS envelope soliton scattering when it propagates along a disordered medium, with rarefied impurities located at random positions. We have shown how strong nonlinearity can provide a mechanism capable to inhibit the localization effects stipulated by the disorder for linear and weakly nonlinear waves. The most remarkable outcome of our calculations is that this effect only takes place when the nonlinear character of solitons exceeds a threshold nonlinearity. This value is given by the nonlinear parameter, i.e. a ratio between the number of quasi-particles in the soliton and its velocity around 1.28505 in proper dimensionless units. Below this threshold, the transmission coefficient tends exponentially to zero as the size of the system increases, either from the beginning (figure 1) or after a short, slower transient (figure 2). The result coincides qualitatively with the ones obtained in $[1,2]$ for the time-independent NLS equation. Above the threshold value our model demonstrates almost undistorted motion of the nonlinear wavepacket along the disordered system, i.e. the transmission coefficient does not decay in the framework of this approximation. A possible reason for the destruction of localization can be the following: as $\alpha$ increases for each fixed value of the initial soliton velocity $V, N$ increases as well and the soliton becomes less extended, as we have already mentioned. We believe that it is more likely that narrow solitons pass unaffected through each one of the delta impurities while, for wide solitons, internal interference effects can appear in the underlying carrier wave, giving rise to localization.

In fact, the effect must be much more complicated. For instance, a rather curious point is that we have found no direct evidence for power law decay behaviour, which was predicted in [1,2]. The main difference between these works and our problem is the modulational instability of the monochromatic nonlinear plane wave, which, in fact, takes place in the model studied in $[1,2]$. In our analytical considerations we used a simple independent scattering approach and Born approximation of the perturbation theory. Of course, the unstable character of this fixed point and the localization decay would have to be proved by direct numerical simulations. We believe that taking into account the additional weak contributions, e.g. interference during the propagation, or direct numerical simulations of the model, will lead to the following picture of nonlinear scattering. The nonlinear transmission coefficient will slowly (probably logarithmically) decay as the size of the system increases, so that the decay length may be presented as the sum of two values, a nonlinear one, $\lambda_{n}$, and a linear localization length, $\lambda_{1}$. The first value essentially depends on the amplitude and, in fact, is very large (it characterizes the decrease of soliton amplitude to a small value which should be much less than unity). The soliton transforms into a linear wavepacket due to scattering along a length of order of $\lambda_{n}$, and then it scatters as a linear object, its transmission coefficient decaying exponentially. Since $\lambda_{n} \gg \lambda_{1}$, the real scattering of nonlinear wavepackets for systems of length $L<\lambda_{n}$ must be very small. A greater effort is needed in order to clarify the real contributions of these neglected effects, as well as to obtain a complete understanding of the underlying basis for the disappearance of localization here reported. 
As the last point, we would like to mention the recent results by Bourbonnais and Maynard [23], who analysed numerically the energy transport in anharmonic lattices with disorder. They found that the regime of diffusion in the nonlinear case is characterized by an exponent which depends strongly on anharmonicity but is insensitive to disorder. That result is in agreement with our prediction of the nonlinear decay length $\lambda_{n}$, above which nonlinear propagation is insensitive to disorder.

\section{Acknowledgments}

We would like to thank A R Bishop, D K Campbell and St Pnevmatikos for discussions of some points of the present paper. All the numerical work was carried out using the IBM 3090 computer at the Centro de Investigaciones Energéticas, Medio Ambientales y Tecnológicas (CIEMAT), Spain, which we gratefully acknowledge. Two of us (A S and L V) are grateful for financial support from the Universidad Complutense de Madrid and the Dirección General de Investigación Científica y Técnica, under grant PB86-0005.

\section{Appendix. Behaviour of $\alpha(z)$ near $\alpha_{c}$}

In this appendix we are going to prove the unstable character of the fixed point $\alpha_{c}$ of equations (39) and (40) that we mentioned above. To this end, let us rewrite that system as

$$
\begin{aligned}
& \frac{\mathrm{d} \alpha}{\mathrm{d} z}=\frac{1}{N^{2}} G_{1}(\alpha) \\
& \frac{\mathrm{d} N}{\mathrm{~d} z}=-\frac{1}{N} G_{2}(\alpha)
\end{aligned}
$$

where

$$
\begin{aligned}
& G_{1}(\alpha) \equiv \frac{\alpha^{2}}{2}\left(\int_{0}^{\infty} \mathrm{d} y y^{2} F(y, \alpha)+\left(\alpha^{2}-3\right) \int_{0}^{\infty} \mathrm{d} y F(y, \alpha)\right) \\
& G_{2}(\alpha) \equiv \alpha \int_{0}^{\infty} \mathrm{d} y F(y, \alpha) .
\end{aligned}
$$

In this new notation $\alpha_{c}$ is nothing but the solution of the equation $G_{1}\left(\alpha_{c}\right)=0$. Now, performing a Taylor expansion of equation (A1) around $\alpha=\alpha_{c}$, we obtain

$$
\frac{\mathrm{d} \alpha}{\mathrm{d} z}=\frac{1}{N^{2}}\left[G_{1}\left(\alpha_{\mathrm{c}}\right)+\left(\alpha-\alpha_{\mathrm{c}}\right)\left(\frac{\mathrm{d} G_{1}}{\mathrm{~d} \alpha}\right)_{\alpha=\alpha_{\mathrm{c}}}+\ldots\right]
$$

and then substitute equation (A2) by

$$
\frac{\mathrm{d} N}{\mathrm{~d} z}=-\frac{1}{N} G_{2}\left(\alpha_{\mathrm{c}}\right)
$$

which will be approximately valid provided we are not too far from $\alpha=\alpha_{c}$. A straightforward calculation is now enough to show that, retaining only first-order terms 
in the equation for $\alpha$, the solution of the system (A5) and (A6) is given by

$$
\begin{aligned}
& \alpha(z)=\alpha_{c}+\left(\alpha(0)-\alpha_{c}\right)\left(1-\frac{2 B}{N^{2}(0)} z\right)^{-A / 2 B} \\
& N^{2}(z)=N^{2}(0)-2 B z,
\end{aligned}
$$

with

$$
A \equiv\left(\frac{\mathrm{d} G_{1}}{\mathrm{~d} \alpha}\right)_{\alpha=\alpha_{\mathrm{c}}}=25.2(4) \quad B \equiv G_{2}\left(\alpha_{\mathrm{c}}\right)=23.4(6) .
$$

The values shown in equation (A9) are numerical estimates, obtained with an accuracy that is enough for our purposes, for we only want to show that both quantities are greater than zero. Now, consider expression (A7) when the initial condition is very close to but greater than $\alpha_{c}$; then, we are left with a solution of the form

$$
\alpha(z)=\alpha_{c}+\frac{c_{1}}{\left(1-c_{2} z\right)^{k}}
$$

and it turns out that, for such a choice of initial condition, $\alpha$ will initially grow, moving away from $\alpha_{c}$, because all the constants $c_{1}, c_{2}$ and $k$ are positive. Very much before $z$ reaches values that make the denominator small, the analysis leading to this equation ceases to be valid, because $\alpha(z)$ is not near $\alpha_{c}$ anymore, so there are no problems of divergence. A similar calculation can be carried out for the initial condition that $\alpha(0)$ is less than but near $\alpha_{c}$; then $c_{1}$ is negative, and $\alpha(z)$ decreases, again diverging from $\alpha_{c}$. To obtain a clearer view of this point, we have plotted in figure 6 some solutions with initial conditions very near $\alpha_{\varepsilon}$, and they look very much the same as those obtained directly from the system (39) and (40). So $\alpha_{c}$ is an unstable fixed point, as we wished to prove.

\section{References}

[1] Devillard P and Souillard B 1986 J. Stat. Phys. 43423

[2] Doucot B and Rammal R Europhys. Lett. 3969 (1987); J. Phys. (Paris) 48509 (1987)

[3] Li, Q, Soukoulis C M, Pnevmatikos St and Economou E N 1988 Phys. Rev. B 3211888

[4] Knapp R, Papanicolaou G and White B 1989 Nonlinearity and Disorder (Springer Proceedings in Physics 39) ed A R Bishop, D K Campbell and St Pnevmatikos (Berlin: Springer)

[5] Pascual P J, Vázquez L, Pnevmatikos St and Bishop A R 1989 Nonlinearity and Disorder (Springer Proceedings in Physics 39) ed A R Bishop, D K Campbell and St Pnevmatikos (Berlin: Springer)

[6] Kivshar Yu S, Gredeskul S A, Sánchez A and Vázquez L 1990 Phys. Rev. Lett. 641693

[7] Gredeskul S A and Kivshar Yu S 1992 Phys. Rep. in press

[8] Lifshitz I M, Gredeskul S A and Pastur L A 1988 Introduction to the Theory of Disordered Systems (New York: Wiley)

[9] Peyrard M and Bishop A R 1990 Nonlinear Coherent Structures (Lecture Notes in Physics 353) ed M Barthes and J Léon (Berlin: Springer)

[10] Kivshar Yu S 1991 Nonlinearity with Disorder (Springer Proceedings in Physics) ed F Kh Abdullaev, A R Bishop and St Pnevmatikos (Berlin: Springer) in press

[11] Caputo J G, Newell A C and Shelley M 1989 Integrable Systems and Applications, (Lecture Notes in Physics 342) ed M Balabane, P Lochak and C Sulem (Berlin: Springer)

[12] Kivshar Yu S and Malomed B A 1989 Rev. Mod. Phys. 61763

[13] Makhankov V. G. 1978 Phys. Rep. 351

[14] Aubry S J. Chem. Phys. 623217 (1975); J. Chem. Phys. 643392 (1976)

[15] Krumhansl J A and Schrieffer J R 1975 Phys. Rev. B 113535 
[16] Jackiw R and Schrieffer J R 1981 Nucl. Phys. B 190 [FS3] 253

[17] Newell A C 1985 Solitons in Mathematics and Physics (Philadelphia: SIAM)

[18] Tappert F and Varma C 1970 Phys. Rev. Lett. 251108

[19] Wu J, Wheatley J, Putterman S and Rudnick I 1987 Phys. Rev. Lett. 592744

[20] Perel V I and Polyakov D G 1984 Zh. Eksp. Teor. Fiz. 86352 (1984 Sov. Phys. JETP 59 204)

[21] Kivshar Yu S and Malomed B A 1985 Phys. Lett. 111A 427

[22] Kivshar Yu S, Kosevich A M and Chubykalo O A 1987 Phys. Lett . 125A 35

[23] Bourbonnais R and Maynard R 1990 Phys. Rev. Lett. 641397 